\title{
Liquid-like pseudoelasticity of sub-10-nm crystalline silver particles
}

\author{
Jun Sun ${ }^{1 \dagger}$, Longbing $\mathrm{He}^{1 \dagger}$, Yu-Chieh $\mathrm{Lo}^{2,3,4 \dagger}$, Tao Xu', Hengchang Biं , Litao Sun ${ }^{1 \star}$, Ze Zhang ${ }^{5 \star}$, \\ Scott X. Mao ${ }^{6}$ and Ju Li ${ }^{2,3 \star}$
}

In nanotechnology, small-volume metals with large surface area are used as electrodes, catalysts, interconnects and antennae $^{1-4}$. Their shape stability at room temperature has, however, been questioned. Using in situ high-resolution transmission electron microscopy, we find that $\mathrm{Ag}$ nanoparticles can be deformed like a liquid droplet but remain highly crystalline in the interior, with no sign of dislocation activity during deformation ${ }^{5,6}$. Surface-diffusion-mediated pseudoelastic deformation is evident at room temperature, which can be driven by either an external force or capillary-energy minimization. Atomistic simulations confirm that such highly unusual Coble pseudoelasticity can indeed happen for sub-10-nm Ag particles at room temperature and at timescales from seconds to months.

Nanotechnology requires precise shape control of components. Once a certain shape is made, it is often hoped that it stays unchanged over a long period of time. Nanoelectronic devices with $14 \mathrm{~nm}$ feature sizes are commercially available at present, and we may soon enter the sub-10-nm regime. It is therefore essential to examine the shape change and shape stability of sub-10-nm material components, especially close to room temperature.

Although both plastic and pseudoelastic deformations are used for shaping, and both incur energy dissipation, the essential difference is that, when unloaded to zero stress, a pseudoelastic component always recovers to a single 'rest shape' regardless of previous deformation history, whereas a component deformed by plasticity has no memory of a single rest shape and will stay in the deformed shape(s) at zero stress (see Supplementary Part 15 for an illustration of this difference). In this paper we will show using in situ high-resolution transmission electron microscopy that sub-10-nm single crystals of Ag deform pseudoelastically, rather than plastically, at room temperature. This victory of pseudoelasticity over plasticity at the small extremes of size-dependent mechanical behaviour of metals ${ }^{5-9}$ can have three profound practical consequences: there is no longer 'plastic freedom' at the small extremes-that is, the availability of a large number of arbitrary, stress-free shapes; one can still gain access to an infinite number of shapes, but different finite loads must be imposed via external constraints, similar to inflating a rubbery balloon; omni-directional, reconfigurable and damage-tolerant contact is suddenly possible at this extreme scale.

\section{Liquid-like deformation of silver nanocrystals}

Figure 1 shows a typical cycle of compression and stretching of a silver (Ag) nanocrystal (NC) with a base diameter of $9.8 \mathrm{~nm}$ and partially bonded on the surface of a W tip. The NC height is $4.6 \mathrm{~nm}$ and the shape is stable under electron-beam irradiation during normal imaging (the beam intensity of $3.8 \mathrm{~A} \mathrm{~cm}^{-2}$ is much weaker than in normal practice) $)^{10,11}$ (Methods and Supplementary Part 1). This shape probably possesses the minimum free energy. The facet shown in Fig. 1a corresponds to the (111) crystal plane of Ag (Supplementary Part 2). As the NC-decorated W tip approaches the crystalline $\mathrm{ZrO}_{2}$ above (Fig. 1b), the top of the Ag NC snaps slightly onto the $\mathrm{ZrO}_{2}$ surface, as a result of van der Waals attraction ${ }^{12}$. The upper part of the Ag NC is then deformed, as can be seen in Fig. 1c. As the $\mathrm{W}$ tip further approaches the $\mathrm{ZrO}_{2}$, compressive stress is spread onto the entire Ag NC. In Fig. 1d-f, the Ag NC undergoes drastic deformation, resulting eventually in a flat pancake-like shape. During the process, the outer morphology of the Ag NC changes similarly to a liquid drop (Supplementary Movies 1-3). However, high-resolution images taken during the process prove that the deforming Ag nanoparticle remains single crystalline (Fig. 1k,l) inside. When the $\mathrm{W}$ tip is detached from the $\mathrm{ZrO}_{2}$ (Fig. 1f-i), the Ag NC surprisingly starts to recover its original shape and the base diameter decreases from 14.3 to $9.5 \mathrm{~nm}$ in the first stage, and then to $9.2 \mathrm{~nm}$. The height of the Ag NC recovered quickly and a thin sharp Ag tip meniscus (Fig. 1i) is formed. When the tip is finally detached, the shape of the Ag NC changes rapidly from an elongated spire to a stable geometry with facets similar to its initial ones (Fig. 1j). Such a reversible change in shape (except for missing atoms induced by effects such as tip scratching and electron bombardment, which break the ideal behaviour) is infinitely repeatable (Fig. 1m,n and Supplementary Movies 1 and 2) just like the deformation of a water droplet, also for lateral squeezing (Supplementary Part 16) and for smaller Ag NCs (Supplementary Movie 4). Thus, the videotaped deformations are pseudoelastic rather than plastic; the memory of a single rest shape can be explained by capillary-energy minimization-that is, the rest shape is the result of the balance between Ag surface energy and $\mathrm{Ag}-\mathrm{W}$ interfacial energy.

\section{Surface-diffusion-dominated Coble pseudoelasticity}

Next we demonstrate that the atomic mechanism behind the shape evolution is surface diffusion. There is prior evidence in the

\footnotetext{
${ }^{1}$ SEU-FEI Nano-Pico Center, Key Lab of MEMS of Ministry of Education, Southeast University, Nanjing, 210096, China, ${ }^{2}$ Department of Nuclear Science and Engineering, Massachusetts Institute of Technology, Cambridge, Massachusetts 02139, USA, ${ }^{3}$ Department of Materials Science and Engineering, Massachusetts Institute of Technology, Cambridge, Massachusetts 02139, USA, ${ }^{4}$ Center for Elements Strategy Initiative for Structural Materials (ESISM), Kyoto University, Sakyo, Kyoto 606-8501, Japan, ${ }^{5}$ Department of Materials Science and Engineering, State Key Laboratory of Silicon Materials, Zhejiang University, Hangzhou 310027, China, ${ }^{6}$ Department of Mechanical Engineering and Materials Science, University of Pittsburgh, 3700 O'Hara Street, Pittsburgh, Pennsylvania 15261, USA. TThese authors contributed equally to this work. *e-mail: slt@seu.edu.cn; zezhang@zju.edu.cn; liju@mit.edu
} 

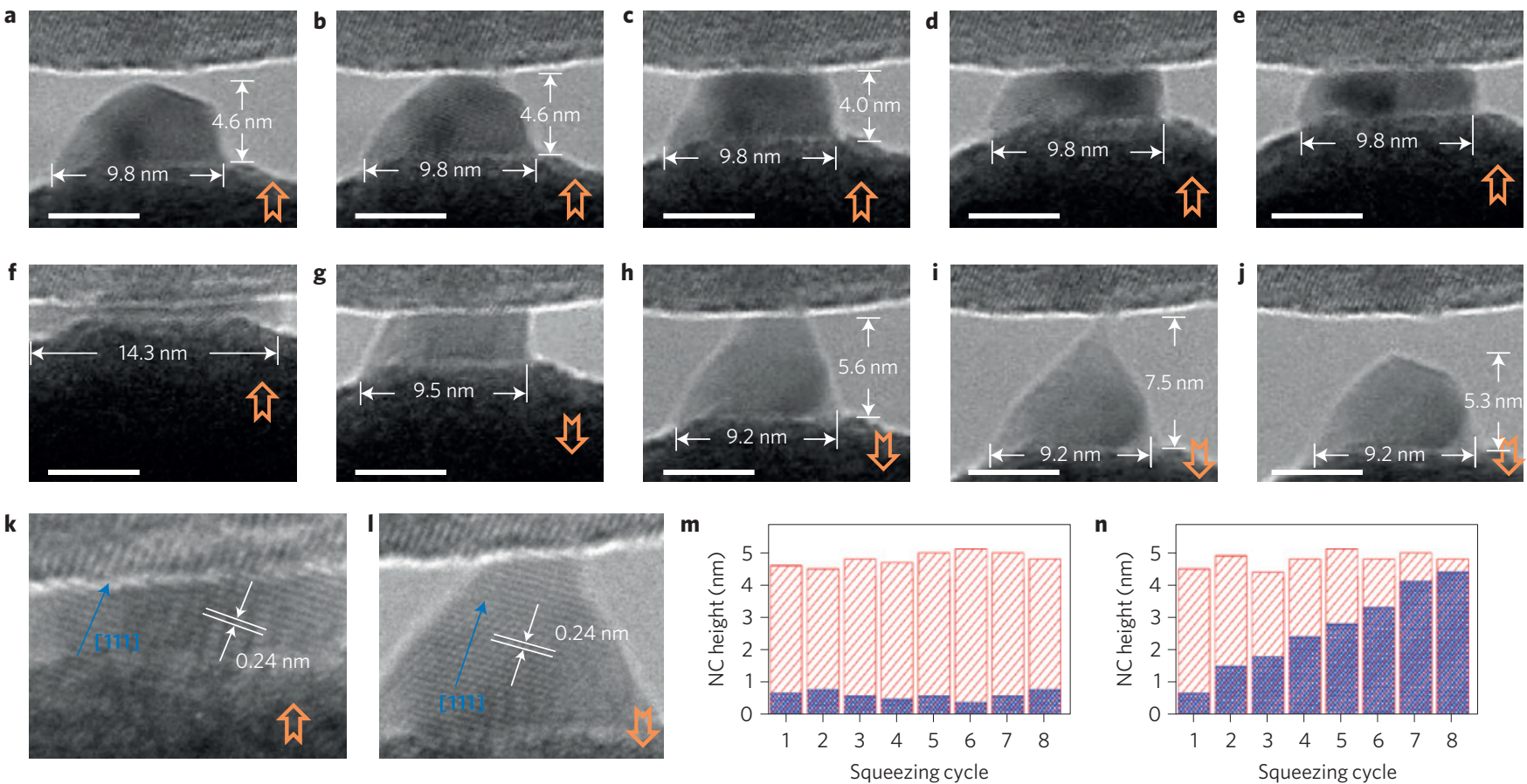

Figure 1 | Reversible pseudoelastic deformation of the Ag NC. a, Initial geometry of the Ag NC. The diameter of the base is $9.8 \mathrm{~nm}$ and the height is $4.6 \mathrm{~nm}$. b-i, Dynamic shape evolution of the Ag NC during compression and stretching. The base part retained its size in the first stages of compression, and later turned to a pancake-like structure. During stretching, the base almost recovered its initial size, with a slight decrease from $9.8 \mathrm{~nm}$ to $9.2 \mathrm{~nm}$. j, Final geometry of the $\mathrm{Ag} \mathrm{NC.} \mathbf{k}, \mathbf{l}, \mathrm{A}$ snapshot of the Ag NC during deformation. The lattice fringes indicate that the NC is crystalline. The crystal orientation is maintained during deformation (blue arrows). The orange arrows indicate the movement direction of the $W$ tip. $\mathbf{m}, \mathbf{n}$, Repetitive compression of the Ag NC to the same (m) and different (n) heights for eight cycles. The NC height in each cycle was measured during (blue columns) and after (red columns) compression. All scale bars, $5 \mathrm{~nm}$.

literature ${ }^{5,6}$ of the dislocation-free diffusional deformation of gold. Here, the imposed stress could be relieved by atom plating/ablation from $\mathrm{Ag}$ surfaces to $\mathrm{Ag}-\mathrm{W}$ or $\mathrm{Ag}-\mathrm{ZrO}_{2}$ interfaces, as in diffusive Coble creep $^{13}$, rather than by displacive dislocation slip, which is normally expected at room temperature ${ }^{14}$. This is evidenced by the direct high-resolution observation of another Ag NC. As shown in Fig. 2, a triangularly shaped Ag NC is compressed gently. Initially, the Ag NC presents a nearly stable geometry during imaging, apart from some flickering sites on the surface steps (Supplementary Movie 5), indicating that the atoms on those sites must be more active diffusively. When the $\mathrm{W}$ tip reaches the $\mathrm{Ag} \mathrm{NC}$, a fresh atomic layer is instantly formed, as marked by the black arrow in Fig. 2b. The new atomic layer grows along the surface when more compression is applied by the W tip (Fig. 2c,d), which clearly cannot be induced by a dislocation slip that would have caused a Burgers-vector-oriented surface step. When the W tip moves further, the whole Ag NC deforms, exhibiting a liquid-like change in morphology (Supplementary Movie 6), with continuous rather than discrete changes of morphology ${ }^{14}$. Simulation of this compression process (Fig. 2e) shows that the diffusion of atoms on free surfaces quickly reconstructs the geometry of the Ag NC, although the electron beam may accelerate this process slightly ${ }^{15}$. We define such deformation behaviour as 'Coble pseudoelasticity', meaning a liquid-like recoverable shape change while the atomic structure inside remains single crystalline. Coble pseudoelasticity has a similar physical origin to Coble creep, which can contribute to the room-temperature plasticity of nanocrystalline metals ${ }^{16}$. However, creep strain is expected to be fully plastic, whereas pseudoelastic strain is expected to be fully recoverable as the applied load returns to zero. Coble pseudoelasticity also differs from conventional pseudoelasticity in that it is not driven by martensitic transformations, but by surface diffusion.
We want to demonstrate that capillary energy plays a critical role in the memory and recall of the single rest shape in Coble pseudoelasticity. Thermodynamically, the surface energy is always there, as used in the Wulff construction (now also balanced by the interfacial energy). But to show that, kinetically, the surface energy is sufficient to drive the shape change of a sub-10-nm Ag $\mathrm{NC}$ at room temperature and timescales of seconds to months, a thin $\mathrm{Ag}$ nanowire is stretched to failure, forming a so-called mechanical break junction ${ }^{17}$ such as that frequently used in molecular-electronics devices. We then observe the subsequent shape recovery at room temperature. As shown in Fig. 3, the diameter of the broken Ag tip is just a few nanometres. The initial size of the monitored area, as marked by the black stripes, is $10 \mathrm{~nm}$ in Fig. 3a. Immediately after the wire is fractured and the break junction forms, it shrinks rapidly in the first few seconds, as shown in Fig. 3a-d. The structure of the base part of the Ag tip remains unchanged, and dislocation activity is not observed ${ }^{5,6}$. However, the free surfaces on the upper part of the Ag tip undergo a continuous (not discrete) shape change, without any external stress, revealing significant capillary action at room temperature. The recession rate of the Ag tip is estimated to be $\sim 1 \mathrm{~nm} \mathrm{~s}^{-1}$ initially (Fig. 3a-c) and then decreases markedly (Supplementary Movie 7), agreeing with the theoretical prediction that the shrinking rate should scale as $\dot{h} \propto R^{-n}$, where $R$ is the radius of curvature and $n$ is a large exponent. According to classical curvature-driven surface diffusion theory, $n=3$ (Supplementary Part 10). But kinetic Monte Carlo (kMC) simulations have revealed $n$ to be as large as 15 at temperatures below the surface roughening transition ${ }^{18}$, owing to facet nucleation controlled kinetics ${ }^{18,19}$ not accounted for by continuous-curvaturedriven surface-diffusion theory. Also, one can show that, when deforming under external mechanical stress $\sigma$, for fixed strain rate $\dot{\varepsilon}$ and temperature, the behaviour is $\sigma \propto R^{n}$, so 'smaller is very much 
a

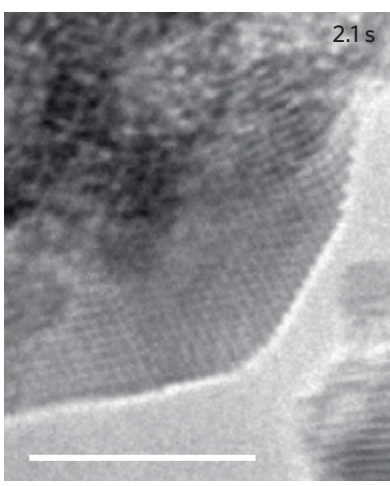

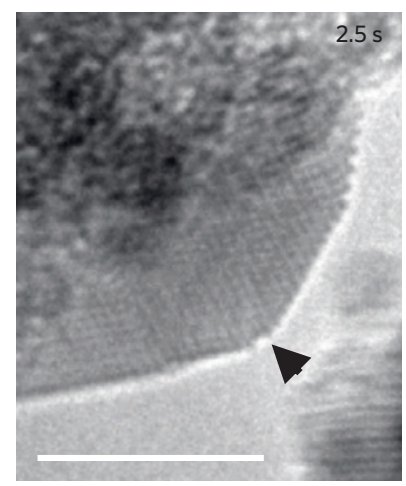
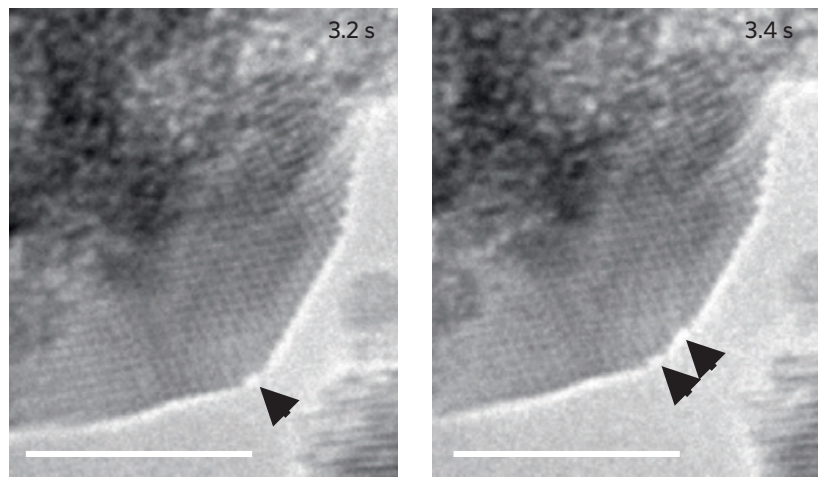

b
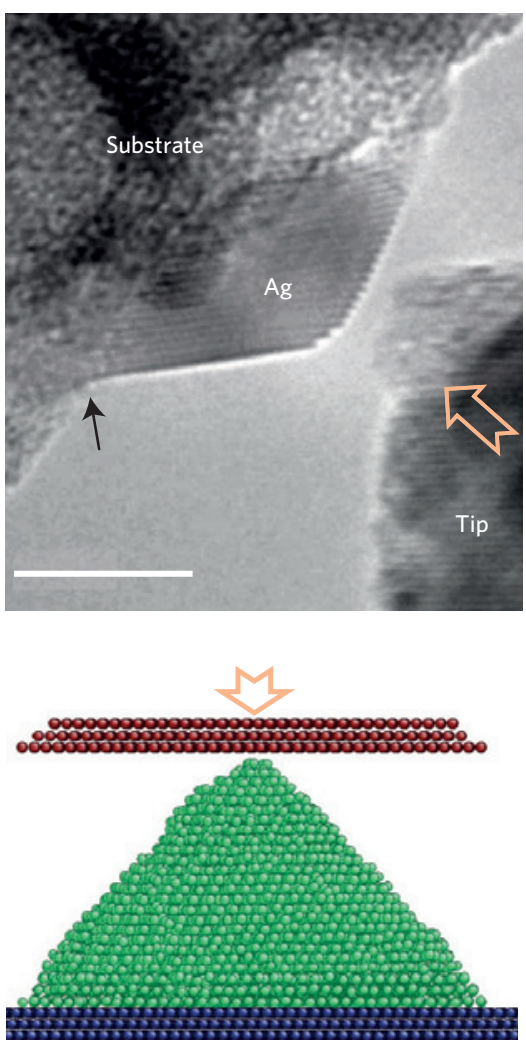

C

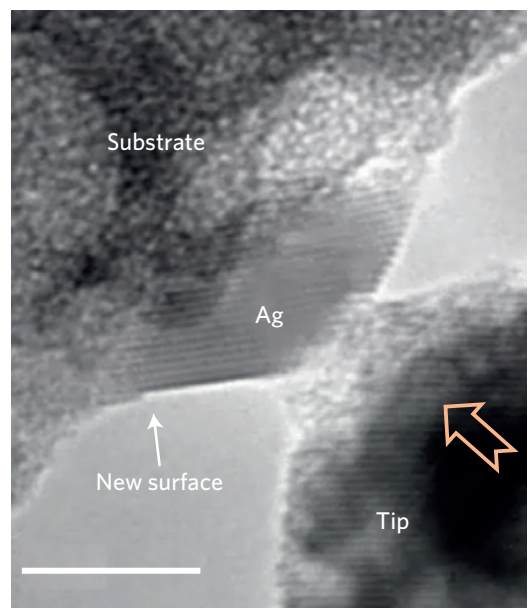

d

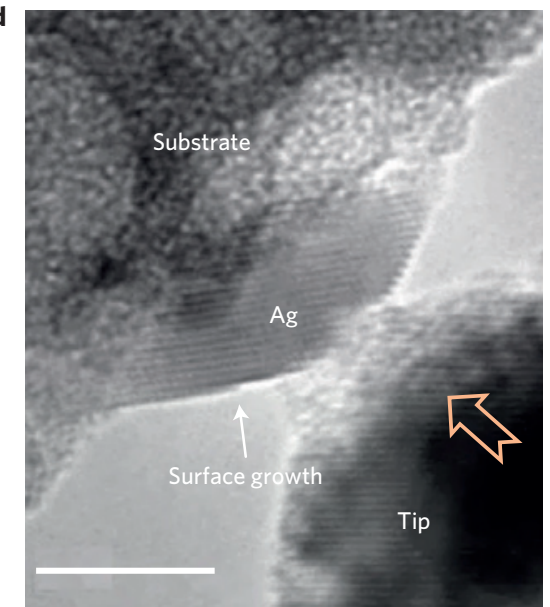

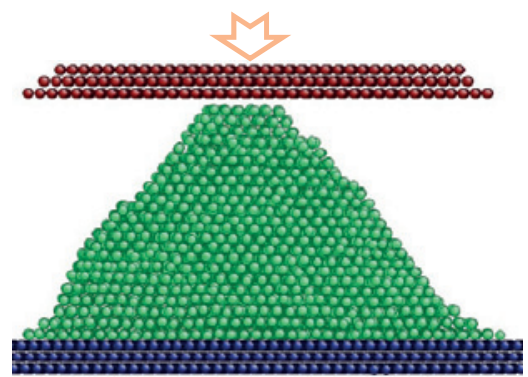

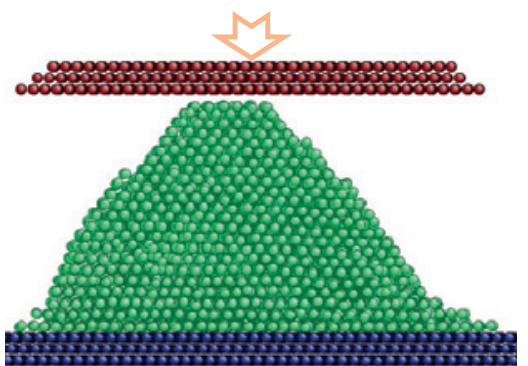

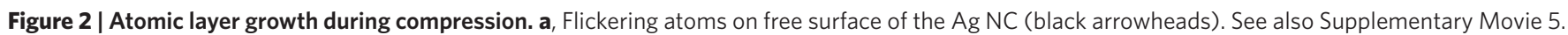
b, Initial geometry of a nanometre-sized Ag NC. c,d, Growth of a fresh atomic layer after gently applying a compressive load. See also Supplementary Movie 6. e, Simulated compression process of a Ag NC. The orange arrows indicate the movement direction of the tip. All scale bars, 5 nm.

weaker' in the Coble deformation regime. The crossover from the Hall-Petch-like 'smaller is stronger' trend $\sigma \propto R^{-\alpha}$ ( $\alpha$ is usually between 0.5 and 1 ) when displacive plasticity is active to the 'smaller is very much weaker' diffusive Coble deformation regime $\sigma \propto R^{n}$ should be sharp and sudden as $R$ is reduced, owing to the large positive exponent $n$. Put simply, we expect the strength to drop precipitously with reducing size in the very small extremes.

Coble pseudoelasticity confirmed by atomistic calculations Inevitably, the imaging electron beam has some effects on the sample ${ }^{20}$. Although we do not expect it to alter the rest shape, electron-beam activation may accelerate surface diffusion (Supplementary Part 14). To ascertain whether Coble pseudoelasticity requires an electron beam to be feasible at room temperature, we perform atomistic calculations to simulate the shape relaxation of a $\mathrm{Ag} \mathrm{NC}$ break junction (Supplementary Movies 8-10). Because of the limited accessible timescale of MD simulations, one strategy is to perform MD at higher temperatures, and then appropriately extrapolate to room temperature. The shape changes of MD simulations at $800 \mathrm{~K}$ for $t_{800 \mathrm{~K}}^{\mathrm{MD}}=0.1 \mu$ s are found to be similar in magnitude and form (Fig. $3 \mathrm{e}-\mathrm{g}$ ) to the TEM observations. Detailed analysis of the atomic trajectories in the $800 \mathrm{~K}$ MD simulations (Supplementary Movie 8) show that: the shape evolution was dominated by single adatom movements, rather than chain or island processes; the shape change was indeed accomplished by atom ablation of the surface layers with greater curvature and by growth of the surface layers with smaller curvature, while atoms two monolayers below the surface always remained highly crystalline; the shape change is rate-controlled by atomic processes on non-(111) facets of the NC, as processes on (111) facets occurred much faster. These findings corroborate a database of activationenergy barriers (summarized in Supplementary Table 1) for FCC metal surface diffusion ${ }^{21,22}$. The ranking of the activation energy 

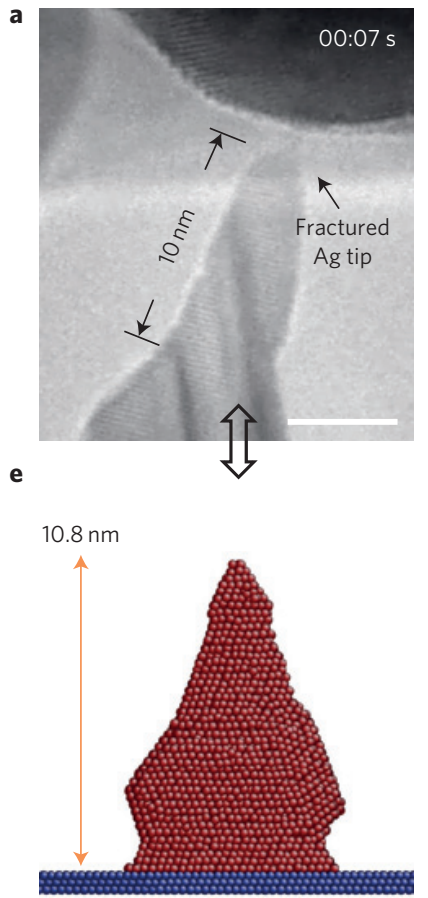
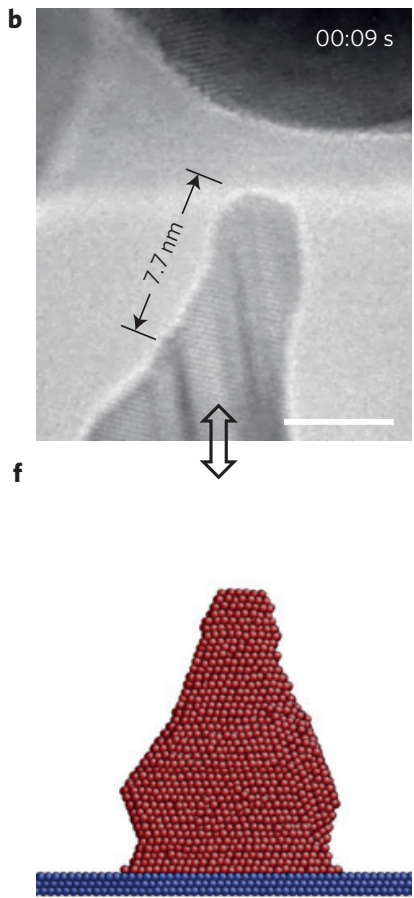

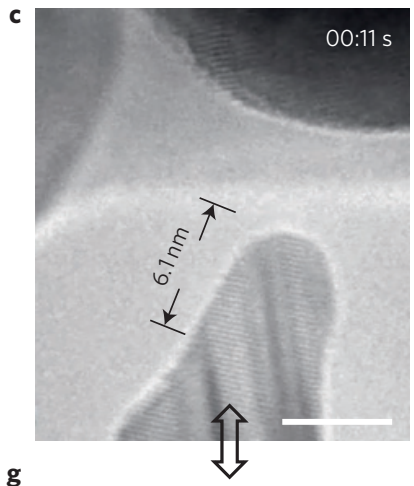

Height of shrinkage $\sim 2 \mathrm{~nm}$

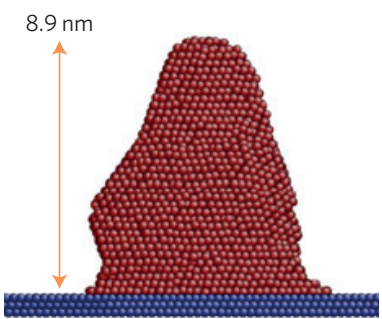

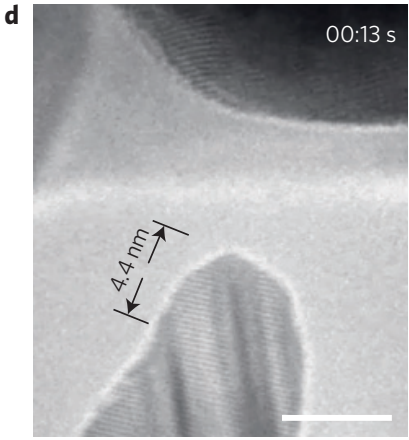

h

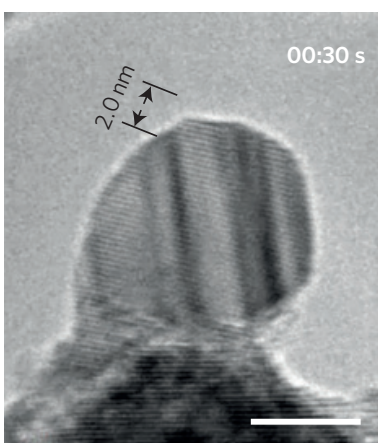

Figure 3 | Surface-energy-driven shape change. a, Initial geometry of the Ag tip after fracture. b-d, Shrinkage process of the Ag tip towards the base part after fracture. $\mathbf{e}-\mathbf{g}$, MD simulations showing that the shrinkage process of the Ag nanoparticle tip is consistent with the experiments in $\mathbf{a}-\mathbf{c}$. $\mathbf{h}, \mathbf{W h e n}$ the radius of curvature of the particle increased, the shrinkage process became slower. All scale bars, $5 \mathrm{~nm}$.

a

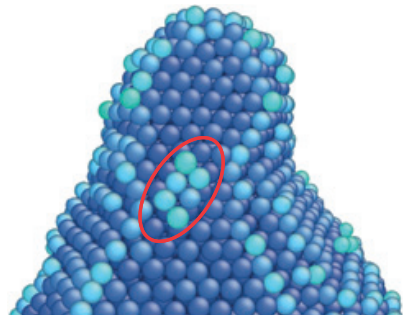

b

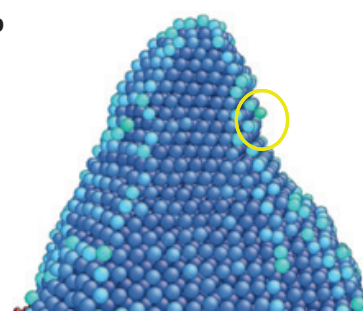

C

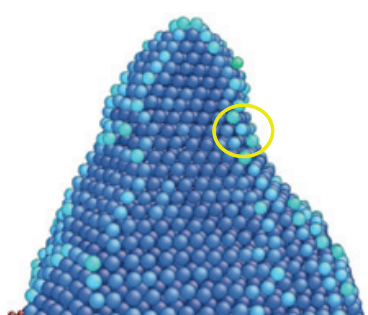

d

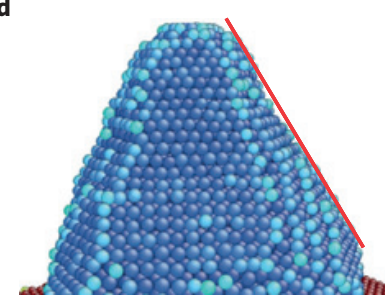

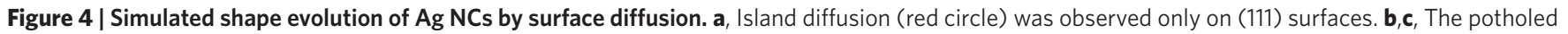
surfaces were smoothed through a series of adatom migration and local reconstruction (yellow circles) on (001) facets. $\mathbf{d}$, After a long time ( $0.1 \mu \mathrm{s})$, the

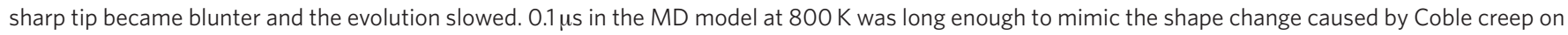
the $\mathrm{Ag} \mathrm{NC}$ at room temperature. The colour gradient represents the difference in potential energy per atom; dark blue represents lower energy and light blue represents higher energy.

barriers of single adatom hopping on different surface facets is: $(110)_{\text {out-channel }}>(001)>(110)_{\text {in-channel }}>(111)$. Atom-chain diffusion was also observed on (111) surfaces (Fig. 4a) and dispersed at the edges with other planes: chain processes were not found on the other facets ${ }^{21}$. The barriers against ascending adatoms are higher than those against descending adatoms, and the barriers for adatoms leaving a corner are higher than for those approaching a corner ${ }^{22}$-thus, high-curvature regions tend to be flattened, a manifestation of the Gibbs-Thomson effect on the chemical potential, which drives the pseudoelasticity. When adatoms arrived at uneven surfaces, they reordered the defective surface by a series of hopping, exchange or pyramid-collapse atomic events, as shown in Supplementary Movie 8 and Fig. 4d. The 'pothole-free' new surface then lowers the barriers for subsequent diffusing atoms $s^{23-26}$ (Fig. 4b,c).

MD simulations also showed that the Ag NC surface mostly consisted of (111) and (001) facets, as well as defective sites near corners or terraces. Many adatoms and small clusters were found on the surface of those facets at the particle tip (Fig. 4). The crystallographic planes on the tip of $10 \mathrm{~nm} \mathrm{Ag}$ nanoparticles coincide with the Wulff construction for $\mathrm{Ag} / \mathrm{Au}$ (refs 27,28). We quantitatively estimated the timescale for room-temperature Coble pseudoelasticity, $\tau$, by applying two newly developed accelerated MD simulation schemes ${ }^{29,30}$ on (001) surface diffusion, which should be rate-controlling in the shape evolution. The distribution of average transition times at different temperatures shows a straight-line behaviour in the Arrhenius plot (Fig. 5). The timescale of diffusion events between 300 and $800 \mathrm{~K}$ shows only a small difference on (111), but varies by $5 \sim 6$ decades for adatom diffusion on the (001) surface, owing to the larger activation energy. The enthalpy of a $10 \mathrm{~nm} \mathrm{Ag}$ particle presents a linear relationship between $300 \mathrm{~K}$ and $800 \mathrm{~K}$ (Supplementary Fig. 4), which suggests no phase transformation in this temperature range. Therefore, most diffusion processes occurring at $800 \mathrm{~K}$ may be extrapolated to $300 \mathrm{~K}$ directly in the Arrhenius fashion. According to the data in Supplemetary Table II, we extrapolated the timescale of the shape change at $300 \mathrm{~K}$ by $t_{800 \mathrm{~K}}^{\mathrm{MD}}(\mathrm{MD}$ simulation time at $800 \mathrm{~K}) \times 10^{5 \sim 6}$ (difference of timescale). Thus, we conclude that the shape evolution or shape relaxation timescale at room temperature should be $\tau=0.01 \sim 0.1 \mathrm{~s}$ for the simulated geometry. 


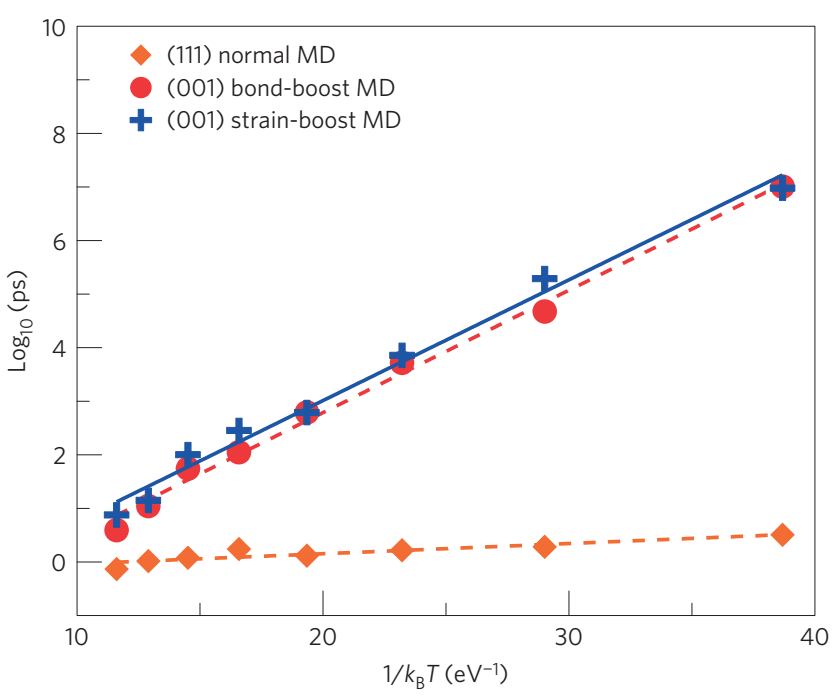

Figure 5 | Arrhenius plots of the transition time for diffusion events on the (001)/(111) surfaces of Ag NCs. Arrhenius plots of the transition time presented as a function of temperature for adatom-diffusion events on (001) and (111) surfaces. We used this Arrhenius relationship to extrapolate the timescale for the Ag-tip shrinkage process at room temperature.

Coble pseudoelasticity (rather than plasticity) is defined by a shape-relaxation time $\tau$ falling below anthropological observation timescales. Our MD and accelerated MD simulations indicate that, even in the absence of an electron beam, Coble pseudoelasticity should occur for sub-10-nm Ag crystals at room temperature. This is corroborated by the following. kMC simulations performed on a generic FCC noble metal showed that $\tau \sim 100 \mathrm{~s}$ for a 1,728-atom nanocrystal ${ }^{18}$ at $T=300 \mathrm{~K}$ (the parameters of the $\mathrm{kMC}$ simulation are not specifically for $\mathrm{Ag}$ ). In a separate experiment, a nanogap was created between two $\sim 20 \mathrm{~nm}$ Au tips, then left for four months, after which it was found that 'The nanogap has clearly expanded, and the locations of greatest curvature have been smoothed out ${ }^{31}$. Given $T_{\text {melt }}(\mathrm{Au})=1,337 \mathrm{~K}>T_{\text {melt }}(\mathrm{Ag})=1,235 \mathrm{~K}$, sub- $10-\mathrm{nm} \mathrm{Ag}$ tips should have $\tau$ much smaller than $20 \mathrm{~nm}$ Au tips. Based on power laws ${ }^{18}$ and experimental data ${ }^{31}$, one can then estimate an upper-bound of $\tau<100 \mathrm{~s}$ for sub-10-nm Ag tips, corroborating the basic conclusion of our simulations that $\tau$ falls near the convenient timeframe of seconds without electron-beam effects. Thus, surface diffusion indeed severely threatens the shape stability of sub-10-nm scale metallic interconnects at room temperature, even for metals with relatively high melting points such as Ag.

\section{Conclusion}

We want to emphasize that although the deformation behaviours are pseudoelastic and 'liquid-like', the nanoparticles have not melted, as the Ag NC remains highly crystalline, and both calculations and previous experimental work ${ }^{5,6,32-36}$ have shown that the melting points of $\mathrm{Ag}, \mathrm{Au}, \mathrm{Al}, \mathrm{Cu}$ and other metals at a size scale of $5 \mathrm{~nm}$ are still very much above room temperature. Thus, the phenomenon we observe is new and distinct from the well-known size-dependent melting point suppression effect ${ }^{32}$. What we see is deformation kinetics, not thermodynamic behaviour of the nanoparticles, and one can see this effect only when one tries to manipulate and follow the nanoparticles in situ (Supplementary Part 13).

In summary, the shape change of sub-10-nm Ag nanoparticles at room temperature is found to be dominated by surface diffusion, making them deform like liquid drops but with a highly crystalline core, which is fundamentally different from displacive shear plasticity by dislocations ${ }^{14}$, and rather unexpected at such a low homologous temperature $\left(T / T_{M}=0.24\right)$. The triumph of pseudoelasticity over plasticity at the small extremes of sizedependent mechanical behaviour of metals can have profound practical consequences on the precise shaping and life estimation of nanometre-scale metallic contacts. It may also open a new avenue for shape-controlling nanodevices with finite forces; for example, the production of omni-directional, reconfigurable and damagetolerant electrical contacts at room temperature, operated using relatively low forces.

\section{Methods}

Fabrication and characterization of Ag NCs. The Ag NCs were formed in situ on a $\mathrm{ZrO}_{2}$ surface by electrically processing a microdevice with a TEM-scanning-tunnelling-microscope $\mathrm{W}$ probe (see Supplementary Fig. 1). The device had a sandwich-like micro-structure $\left(\mathrm{ZrO}_{2}-\mathrm{Ag}-\mathrm{ZrO}_{2}\right)$ and was prepared by gas-phase deposition. The structural evolution was characterized by TECNAI 20 TEM and aberration-corrected TEM (Titan 80-300) with video recorded at 10 frames s ${ }^{-1}$. Compression and stretching cycles of the Ag NCs were conducted at a speed of approximately $0.1 \mathrm{~nm} \mathrm{~s}^{-1}$. During forcible squeezing, the movement speed was approximately $1 \mathrm{~nm} \mathrm{~s}^{-1}$.

MD and accelerated MD simulations. MD simulations were performed using the LAMMPS program ${ }^{37}$. The embedded-atom method (EAM) potential ${ }^{38}$ was applied to describe the interactions between Ag atoms. A flat (111) plane indenter was used to compress and stretch the Ag particles. The interaction force between the indenter and particles was a Lennard-Jones potential with $\varepsilon=0.18 \mathrm{eV}$ and $\sigma=3.49 \mathrm{~A}$. After stretching, the sharp-tipped Ag particle was relaxed at $300,800,900$, and $1,000 \mathrm{~K}$, respectively, for $0.1 \mu$ s to observe the shape evolution. In the accelerated MD simulations, a six-layer slab with 72 atoms in each layer was established, and an adatom was put on the top of (001) surface. The two bottom layers were fixed and the others were mobile. Only the atoms in top two layers (including the adatom) were tagged for boosting by the strain-boost ${ }^{29}$ and bond-boost ${ }^{30}$ methods. The Nose-Hoover NVT ensemble was applied to both MD and accelerated MD. The transition time obtained from accelerated MD at each temperature was the average of ten samplings, which include hopping, exchanging and multiple exchanging events. The parameters of accelerated $\mathrm{MD}$ are $0.4 \mathrm{eV}$ for the boost potential and 0.3 for the boost threshold ${ }^{29}$. Both values are chosen very conservatively after many tests.

Received 26 November 2013; accepted 4 September 2014; published online 12 October 2014

\section{References}

1. Park, M. et al. Highly stretchable electric circuits from a composite material of silver nanoparticles and elastomeric fibres. Nature Nanotech. 7, 803-809 (2012).

2. Chen, J. Y., Lim, B., Lee, E. P. \& Xia, Y. N. Shape-controlled synthesis of platinum nanocrystals for catalytic and electrocatalytic applications. Nano Today 4, 81-95 (2009)

3. Liu, N., Tang, M. L., Hentschel, M., Giessen, H. \& Alivisatos, A. P. Nanoantenna-enhanced gas sensing in a single tailored nanofocus. Nature Mater. 10, 631-636 (2011).

4. Muhlschlegel, P., Eisler, H. J., Martin, O. J. F., Hecht, B. \& Pohl, D. W. Resonant optical antennas. Science 308, 1607-1609 (2005).

5. Rodrigues, V., Fuhrer, T. \& Ugarte, D. Signature of atomic structure in the quantum conductance of gold nanowires. Phys. Rev. Lett. 85, 4124-4127 (2000).

6. Rodrigues, V. \& Ugarte, D. Real-time imaging of atomistic process in one-atom-thick metal junctions. Phys. Rev. B 63, 073405 (2001).

7. Uchic, M. D., Dimiduk, D. M., Florando, J. N. \& Nix, W. D. Sample dimensions influence strength and crystal plasticity. Science 305, 986-989 (2004).

8. Yu, Q. et al. Strong crystal size effect on deformation twinning. Nature 463, 335-338 (2010).

9. Brinckmann, S., Kim, J. Y. \& Greer, J. R. Fundamental differences in mechanical behavior between two types of crystals at the nanoscale. Phys. Rev. Lett. 100, 155502 (2008)

10. Iijima, S. \& Ichihashi, T. Structural instability of ultrafine particles of metals. Phys. Rev. Lett. 56, 616-619 (1986).

11. Wang, Z. W. \& Palmer, R. E. Determination of the ground-state atomic structures of size-selected Au nanoclusters by electron-beam-induced transformation. Phys. Rev. Lett. 108, 245502 (2012).

12. Mordehai, D., Rabkin, E. \& Srolovitz, D. J. Pseudoelastic deformation during nanoscale adhesive contact formation. Phys. Rev. Lett. 107, 096101 (2011).

13. Coble, R. L. A model for boundary diffusion controlled creep in polycrystalline materials. J. Appl. Phys. 34, 1679-1682 (1963).

14. Zheng, H. et al. Discrete plasticity in sub-10-nm-sized gold crystals. Nature Commun. 1, 144 (2010). 
15. Wang, Z. W. \& Palmer, R. E. Mass spectrometry and dynamics of gold adatoms observed on the surface of size-selected Au nanoclusters. Nano Lett. 12, 91-95 (2012).

16. Gleiter, H. Nanocrystalline materials. Prog. Mater. Sci. 33, 223-315 (1989).

17. Reed, M. A., Zhou, C., Muller, C. J., Burgin, T. P. \& Tour, J. M. Conductance of a molecular junction. Science 278, 252-254 (1997).

18. Combe, N., Jensen, P. \& Pimpinelli, A. Changing shapes in the nanoworld. Phys. Rev. Lett. 85, 110-113 (2000).

19. Mullins, W. W. \& Rohrer, G. S. Nucleation barrier for volume-conserving shape changes of faceted crystals. J. Am. Ceram. Soc. 83, 214-216 (2000).

20. Egerton, R. F., Li, P. \& Malac, M. Radiation damage in the TEM and SEM Micron 35, 399-409 (2004).

21. Kim, S. Y., Lee, I. H. \& Jun, S. Transition-pathway models of atomic diffusion on fcc metal surfaces. I. Flat surfaces. Phys. Rev. B 76, 245407 (2007).

22. Kim, S. Y., Lee, I. H. \& Jun, S. Transition-pathway models of atomic diffusion on fcc metal surfaces. II. Stepped surfaces. Phys. Rev. B 76, 245408 (2007).

23. Aminpour, M., Trushin, O. \& Rahman, T. S. Effect of misfit dislocation on surface diffusion. Phys. Rev. B 84, 035455 (2011).

24. Ala-Nissila, T., Ferrando, R. \& Ying, S. C. Collective and single particle diffusion on surfaces. Adv. Phys. 51, 949-1078 (2002).

25. Yildirim, H., Kara, A. \& Rahman, T. S. Origin of quasi-constant pre-exponential factors for adatom diffusion on $\mathrm{Cu}$ and $\mathrm{Ag}$ surfaces. Phys. Rev. B 76, 165421 (2007).

26. Yildirim, H. \& Rahman, T. S. Diffusion barriers for Ag and $\mathrm{Cu}$ adatoms on the terraces and step edges on $\mathrm{Cu}(100)$ and $\mathrm{Ag}(100)$ : $\mathrm{An}$ ab initio study. Phys. Rev. B 80, 235413 (2009).

27. Wang, B. Y., Liu, M. X., Wang, Y. T. \& Chen, X. S. Structures and energetics of silver and gold nanoparticles. J. Phys. Chem. C 115, 11374-11381 (2011).

28. Marks, L. D. Experimental studies of small-particle structrures. Rep. Prog. Phys. 57, 603-649 (1994).

29. Hara, S. \& Li, J. Adaptive strain-boost hyperdynamics simulations of stress-driven atomic processes. Phys. Rev. B 82, 184114 (2010).

30. Miron, R. A. \& Fichthorn, K. A. Accelerated molecular dynamics with the bond-boost method. J. Chem. Phys. 119, 6210-6216 (2003).

31. Strachan, D. R. et al. Clean electromigrated nanogaps imaged by transmission electron microscopy. Nano Lett. 6, 441-444 (2006).

32. Couchman, P. R. \& Jesser, W. A. Thermodynamic theory of size dependence of melting temperature in metals. Nature 269, 481-483 (1977).
33. Lai, S. L., Carlsson, J. R. A. \& Allen, L. H. Melting point depression of Al clusters generated during the early stages of film growth: Nanocalorimetry measurements. Appl. Phys. Lett. 72, 1098-1100 (1998).

34. Bachels, T., Guntherodt, H. J. \& Schafer, R. Melting of isolated tin nanoparticles. Phys. Rev. Lett. 85, 1250-1253 (2000).

35. Asoro, M. A., Damiano, J. \& Ferreira, P. J. Size effects on the melting temperature of silver nanoparticles: In-situ TEM observations. Microsc. Microanal. 15, 706-707 (2009).

36. Lu, H. M., Li, P. Y., Cao, Z. H. \& Meng, X. K. Size-, shape-, and dimensionality-dependent melting temperatures of nanocrystals. J. Phys. Chem. C 113, 7598-7602 (2009).

37. Plimpton, S. Fast parallel algorithms for short-range molecular dynamics. J. Comput. Phys. 117, 1-19 (1995).

38. Sheng, H. W., Kramer, M. J., Cadien, A., Fujita, T. \& Chen, M. W. Highly optimized embedded-atom-method potentials for fourteen fcc metals. Phys. Rev. B 83, 134118 (2011).

\section{Acknowledgements}

This work was supported by the National Basic Research Program of China under gran Nos. 2011CB707601 and 2012CB619402, the National Natural Science Foundation of China under grant Nos. 61274114, 113279028, 51201032 and 51071044, the Key Grant Project of Chinese Ministry of Education under grant No. 311019, and the Natural Science Foundation of Jiangsu Province under grant Nos. BK2011592 and BK2012024. Y-C.L. thanks E. Bitzek (Friedrich-Alexander-Universität Erlangen-Nürnberg) and J. C. Huang (National Sun Yat-Sen University) for kind support. J.L. and Y-C.L. would like to acknowledge support from NSF DMR-1120901 and DMR-1240933. Computational time on the Extreme Science and Engineering Discovery Environment (XSEDE) under grant TG-DMR130038, TG-DMR140003 and TG-PHY140014 is gratefully acknowledged.

\section{Author contributions}

L.S., J.L. and Z.Z. proposed and supervised the project, J.S., L.H., T.X. and H.B. performed the experiments, and Y-C.L. performed the simulations. L.S., J.L., Z.Z. and S.X.M. analysed data and wrote the manuscript. All the authors participated in discussions of the research.

\section{Additional information}

Supplementary information is available in the online version of the paper. Reprints and permissions information is available online at www.nature.com/reprints.

Correspondence and requests for materials should be addressed to L.S., Z.Z. or J.L.

\section{Competing financial interests}

The authors declare no competing financial interests. 